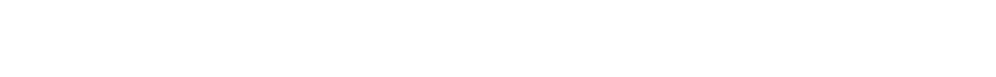

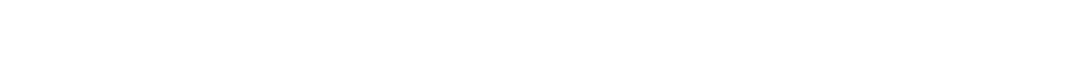

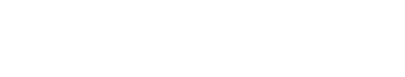

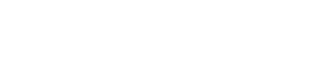

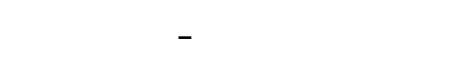

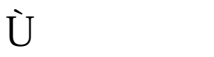

\section{ŭ Ť́:E}

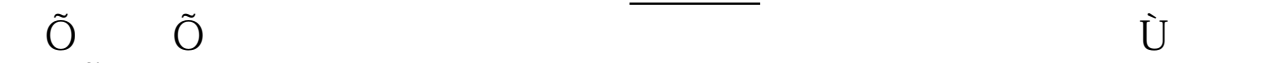

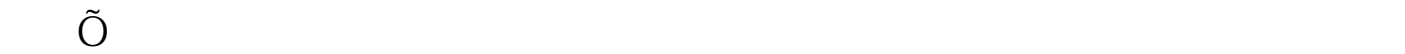

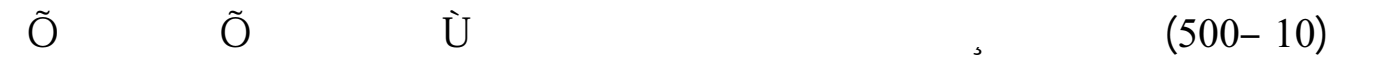

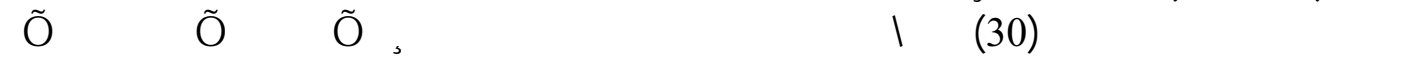

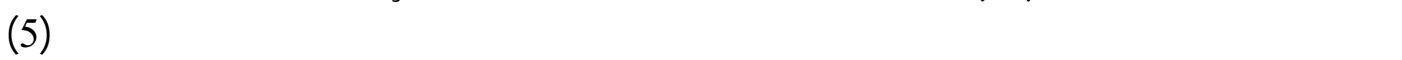

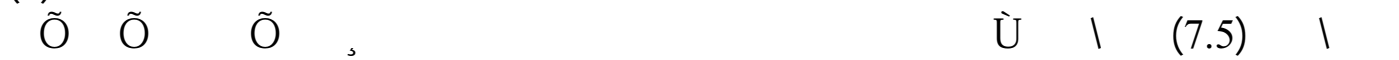

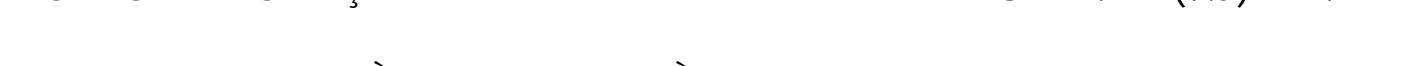

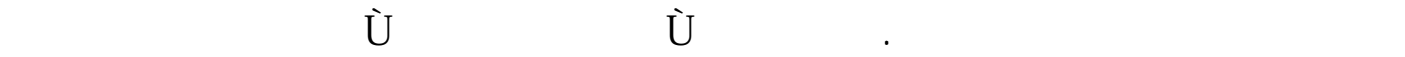

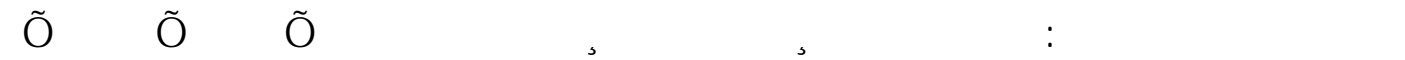

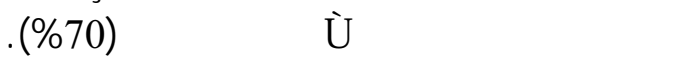

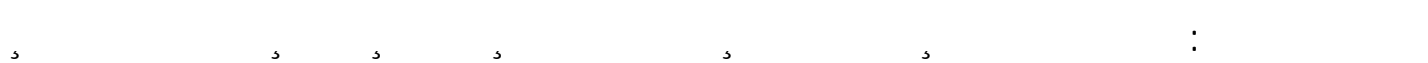

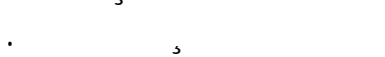

\author{
Use Of Bentonite Clay As A Coagulant Or A Coagulant Aid \\ With Ferrous Sulfate For Synthetic Turbidity Removal \\ Mohammad S. Hassan Musa'b A. Al- Tamir \\ Civil Engg. Dept./University of Mosul
}

\begin{abstract}
In this study the bentonite clay has been used as a coagulant and coagulant aid with ferrous sulfate in turbidity removal for different initial synthetic turbidity of Tigris River samples of (10-500) ntu, The study revealed the efficiency of using bentonite clay as a coagulant alone in removing turbidity with a dose not more than $30 \mathrm{mg} / \mathrm{l}$, Also the study reveled the improvement of ferrous sulfate efficiency for removing water turbidity when bentonite clay used with it in a dose of $7.5 \mathrm{mg} / \mathrm{l}$ of bentonite and $5 \mathrm{mg} / \mathrm{l}$ of ferrous sulfate. The regression analysis showed that the most important variables affecting on turbidity removal were initial turbidity followed by settling time and bentonite clay dose then finally ferrous sulfate dose.
\end{abstract}

Key Words: Ferrous Sulfate, Iron Salts, Bentonite Clay, Coagulation, flocculation, Coagulant aids, Turbidity removal, Water Supply. 


\section{ritsie}

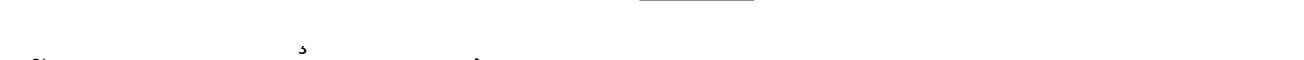

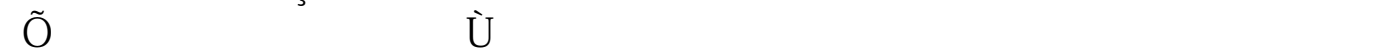

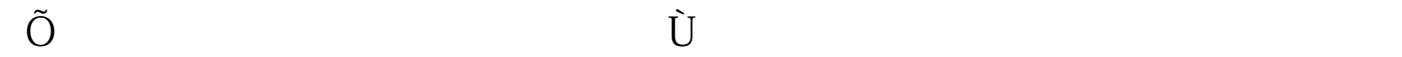
ŠUtC̄É

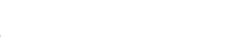

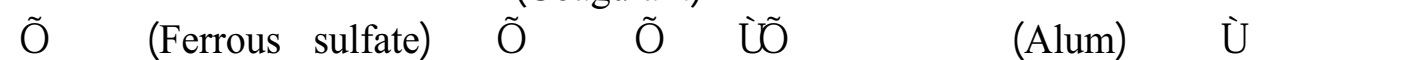

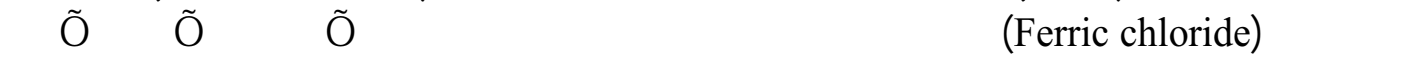

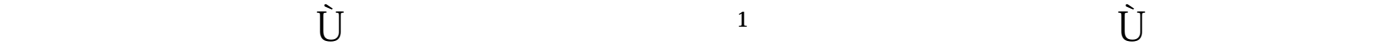

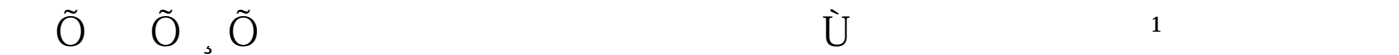

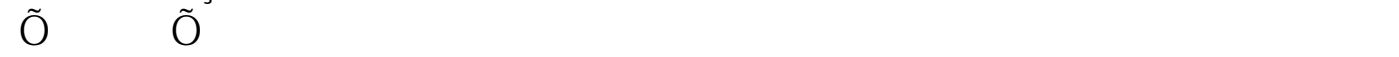

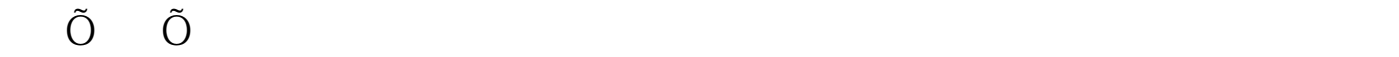

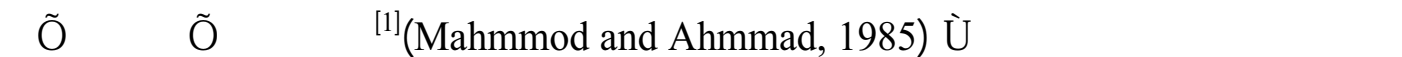

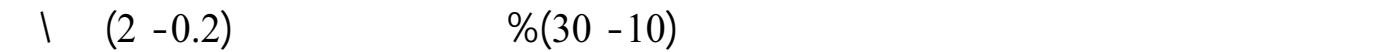

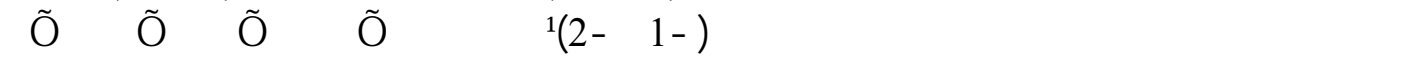

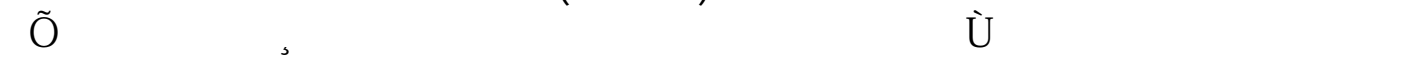

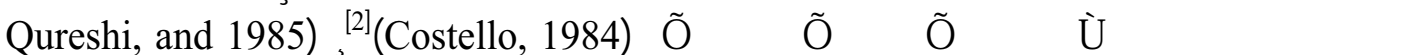

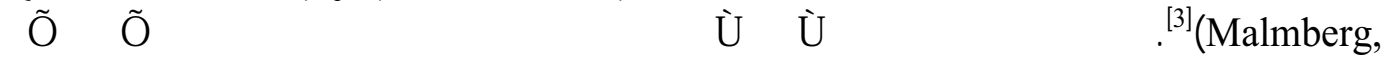

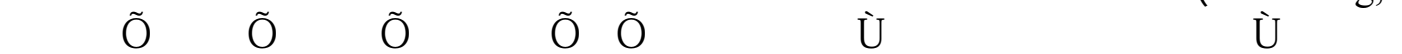

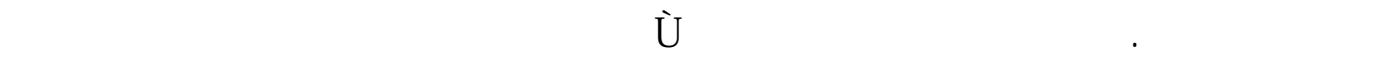

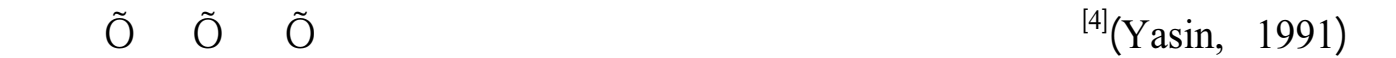

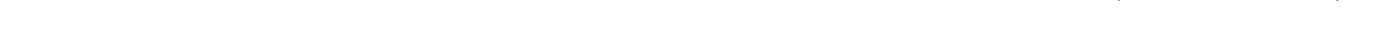

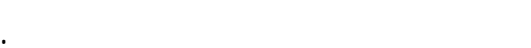

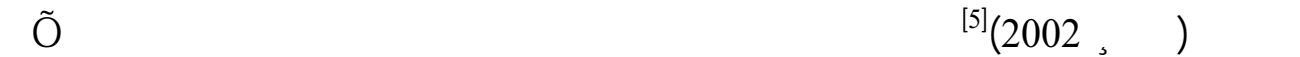

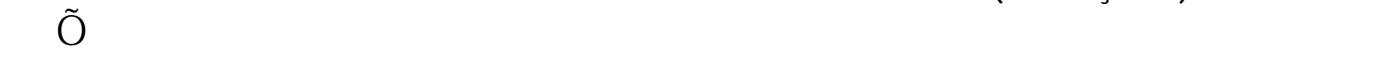

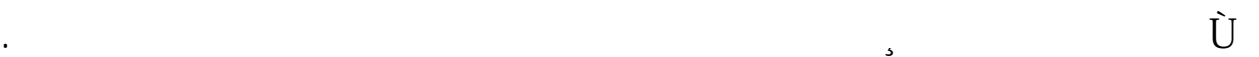

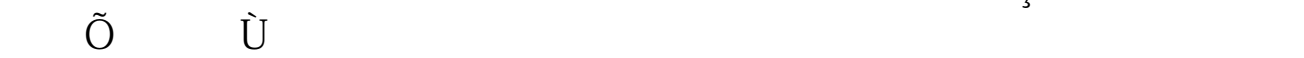

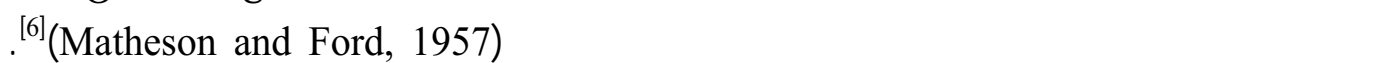
${ }^{[8]}$ (York and Morgan, 1974) $\partial^{[7]}$ (Lund and Nissen, 1986)

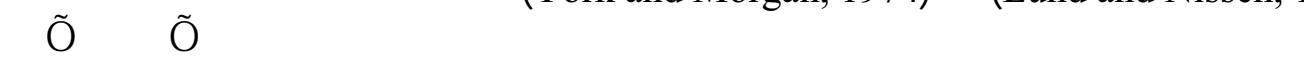

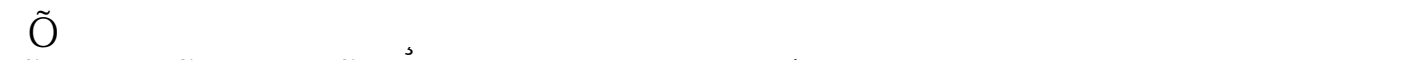

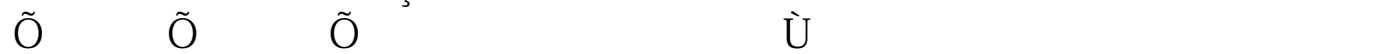

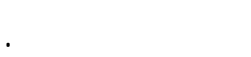

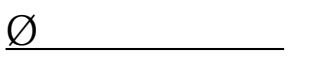

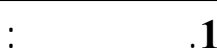

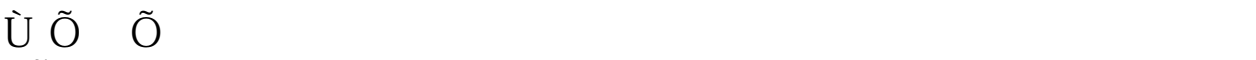

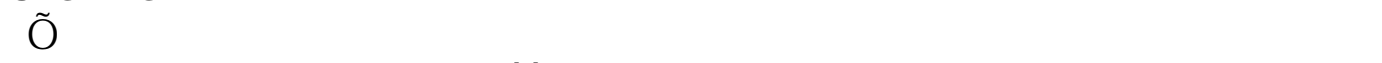

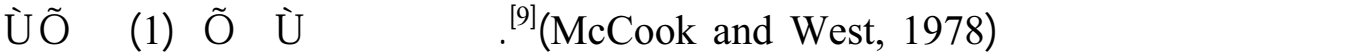

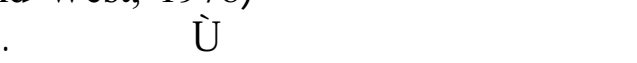


حسن : استخدام طين البنتونايت كمخثر أو كمساعد للتخثير مع كبريتات الحديدوز في إزالة العكورة

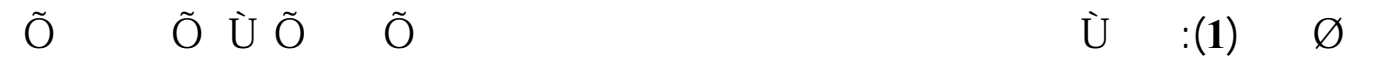
.(1998)

\begin{tabular}{|c|c|c|c|c|}
\hline 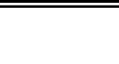 & tgி & $d_{\bar{d}} \overline{j c}$ & tச் & 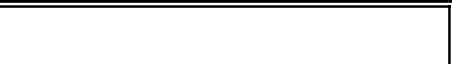 \\
\hline 3.2 & 3.6 & 9.6 & 9 & 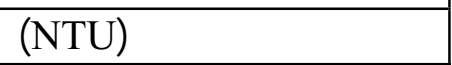 \\
\hline
\end{tabular}

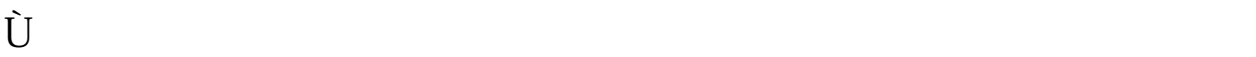

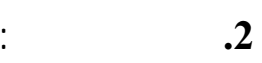

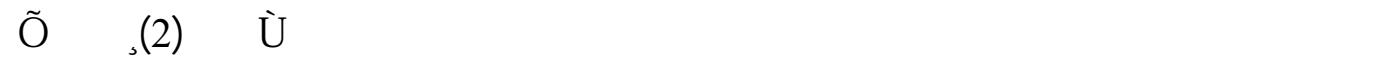

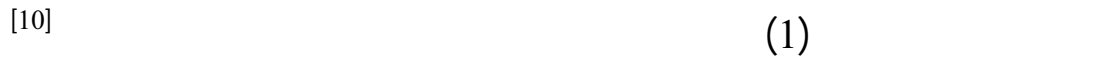

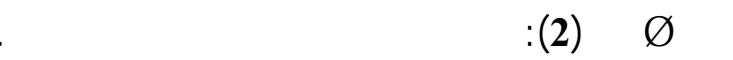

\begin{tabular}{|c|c|c|c|c|c|}
\hline \multicolumn{5}{|c|}{ Sิtghtret } & \multirow{2}{*}{ rfơă } \\
\hline 5 & 4 & 3 & 2 & 1 & \\
\hline 128 & 64 & 61 & 84 & 440 & 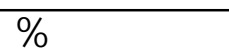 \\
\hline 87 & 45 & 46 & 46 & 97 & 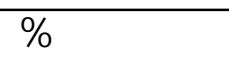 \\
\hline 41 & 19 & 15 & 38 & 343 & 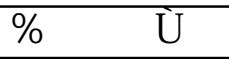 \\
\hline 2.60 & 2.74 & 2.57 & 2.70 & 2.84 & 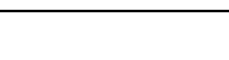 \\
\hline
\end{tabular}

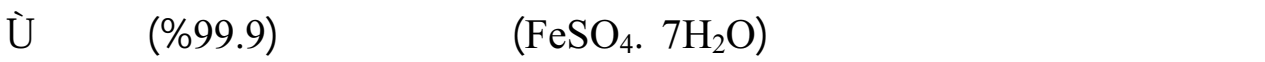

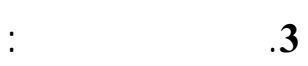

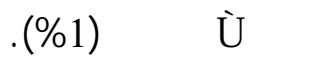

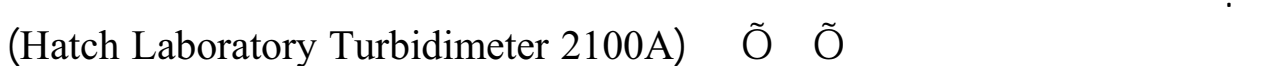

:R̃̃oşh

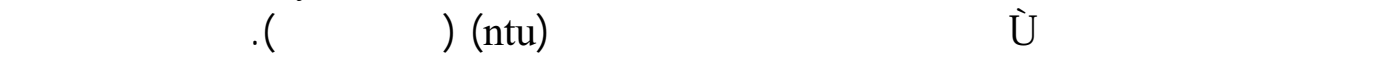

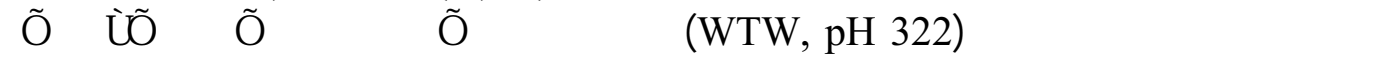

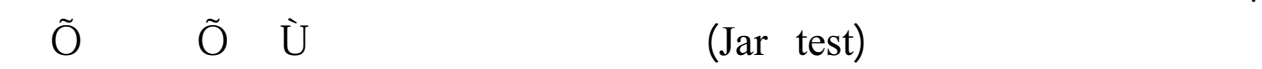
.řð̛̛̃́dŔ

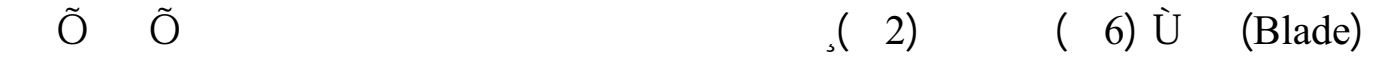

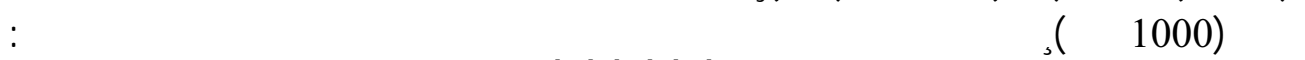

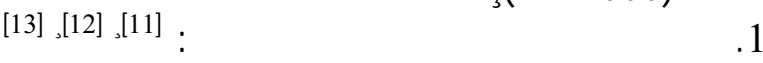

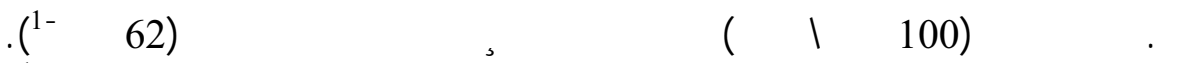

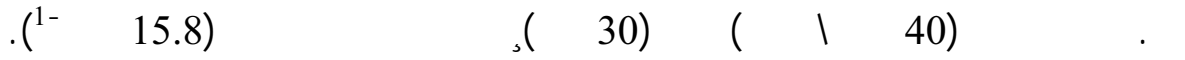

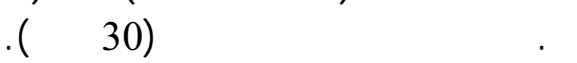

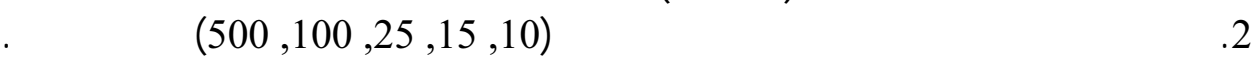

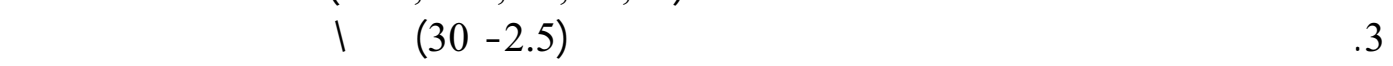
.ř

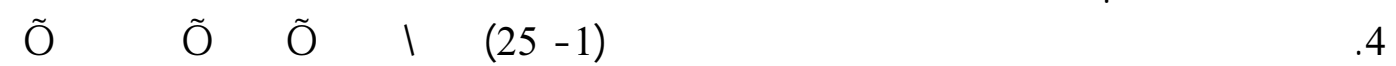

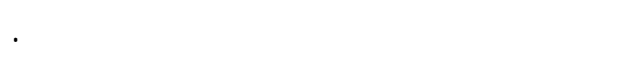

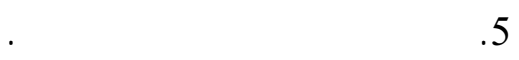

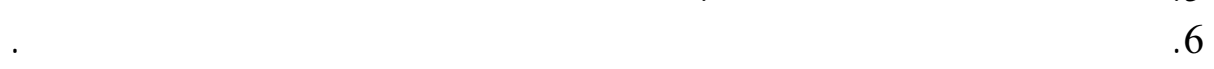




\begin{tabular}{llll} 
Al-Rafidain Engineering & Vol.14 & No.4 & 2006 \\
\hline
\end{tabular}

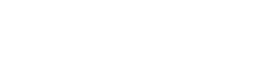

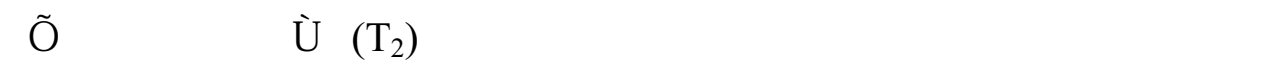

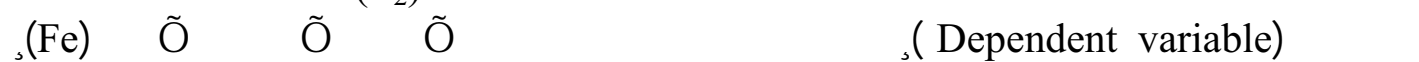

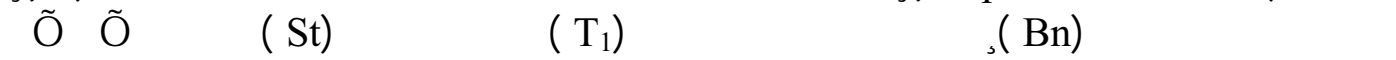

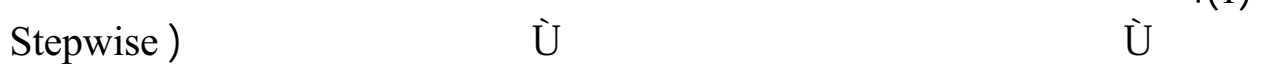

(1) CBi ¿

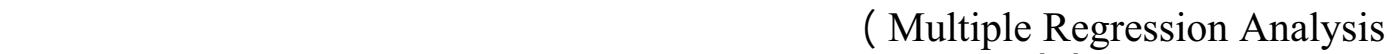

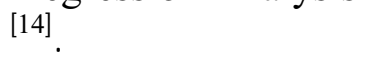

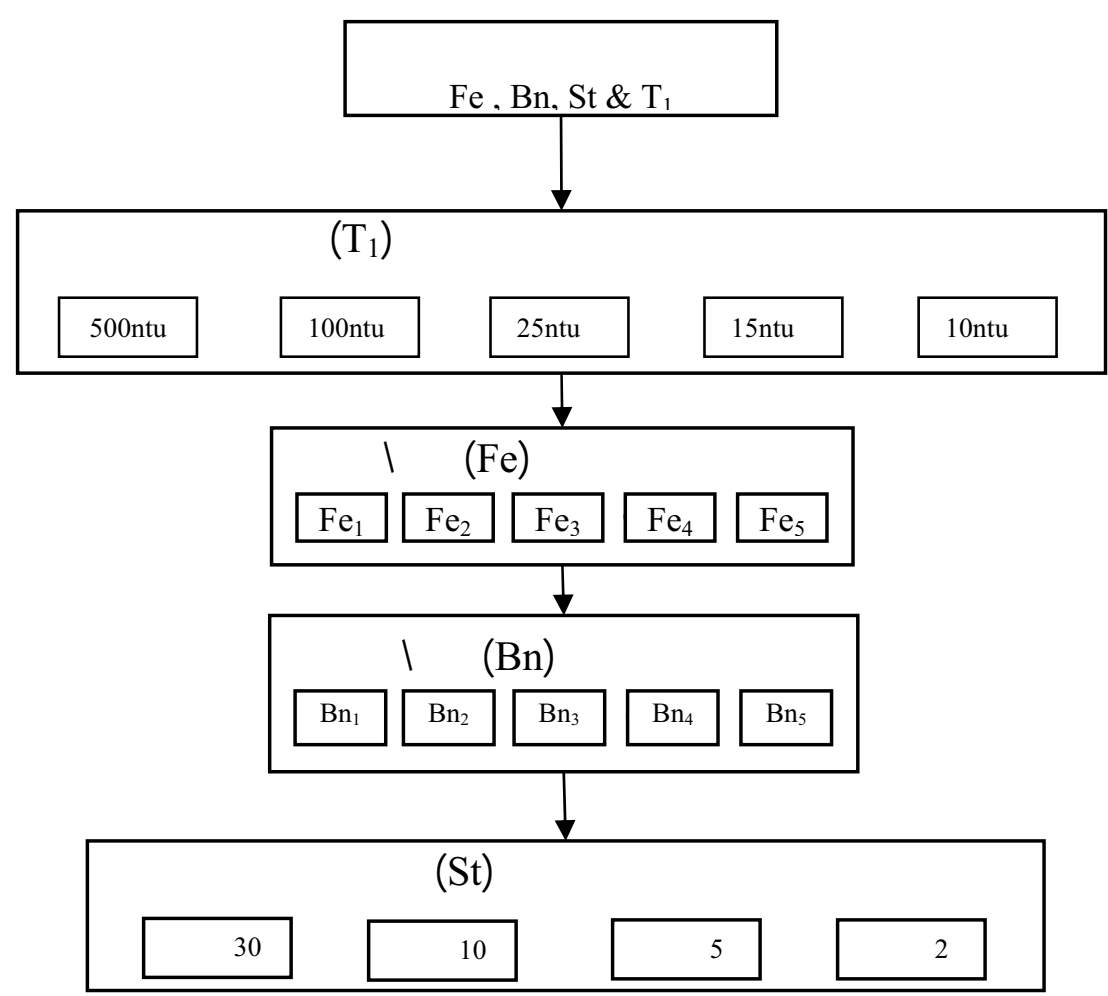

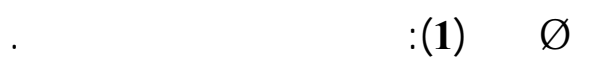




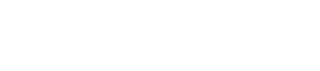

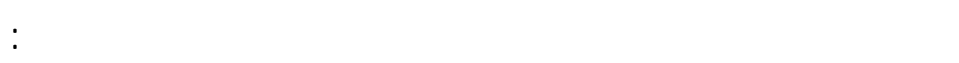

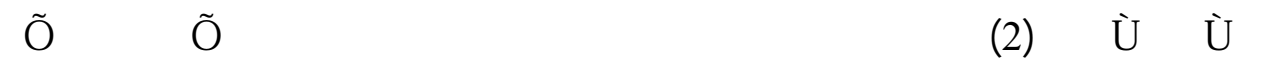

ř» Yิũ

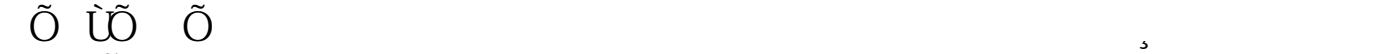

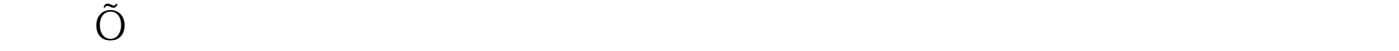

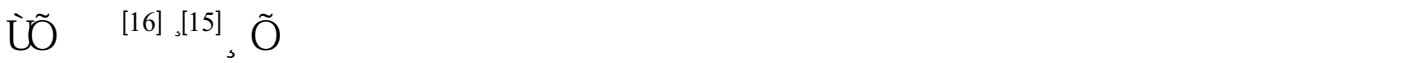

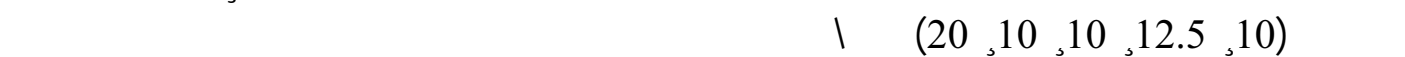

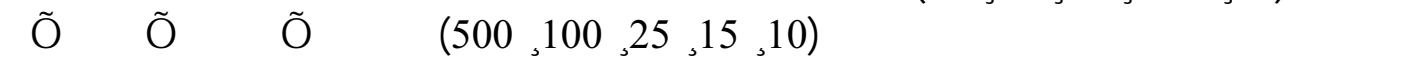

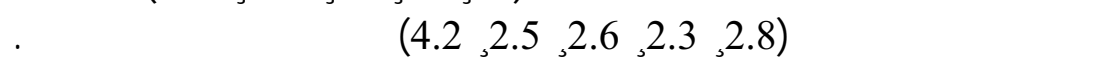

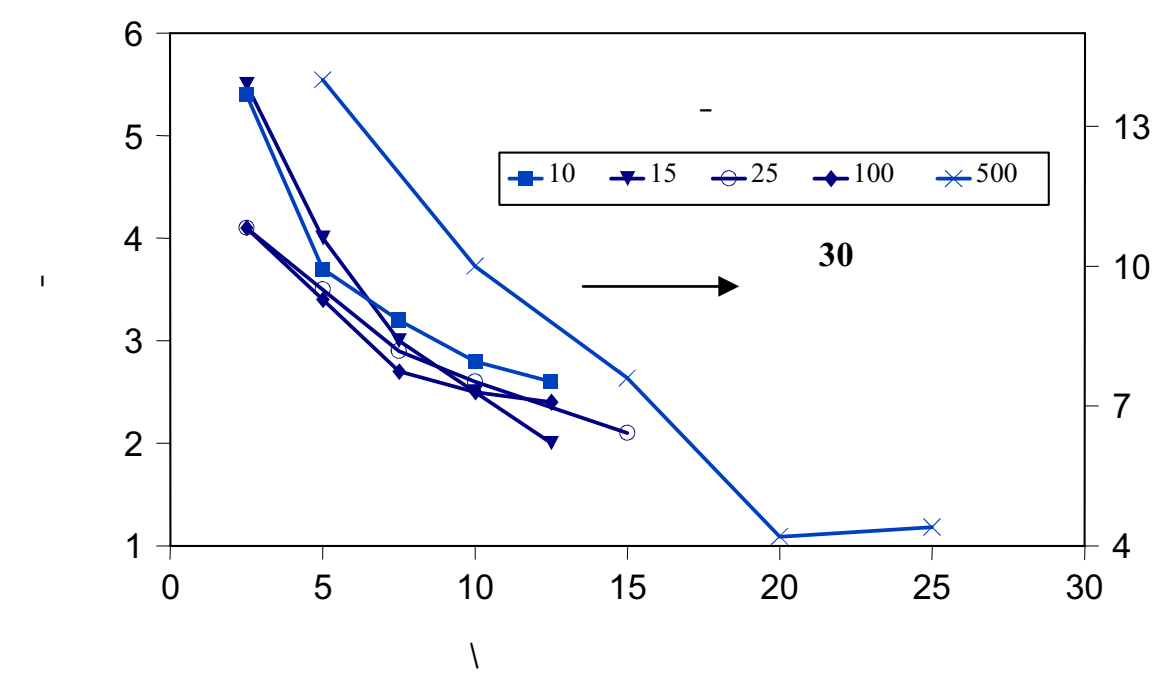

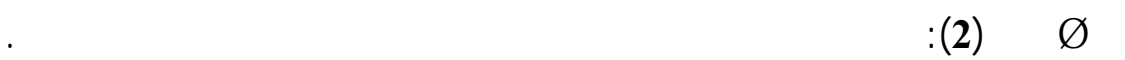

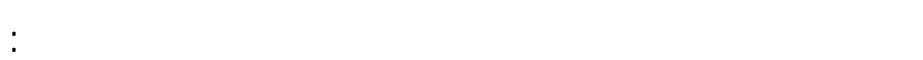

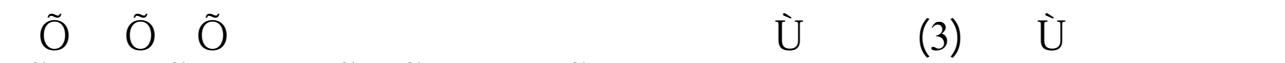

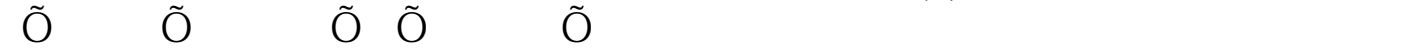

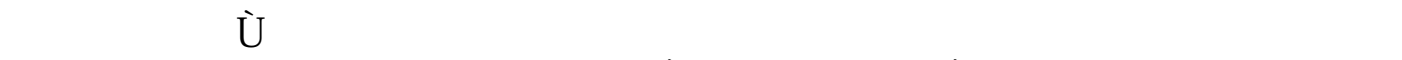

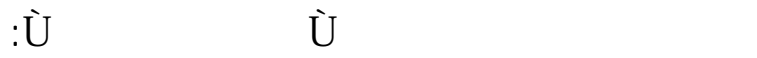

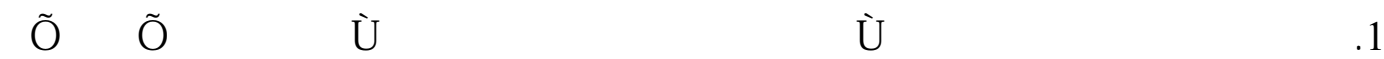

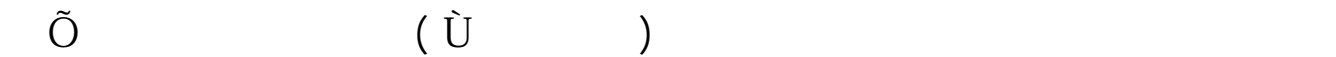

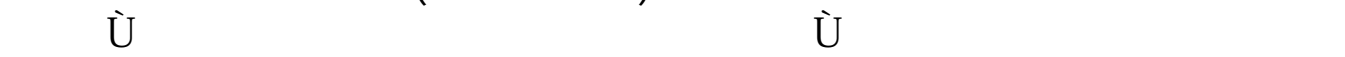
. F̂Ŷ̀ ř

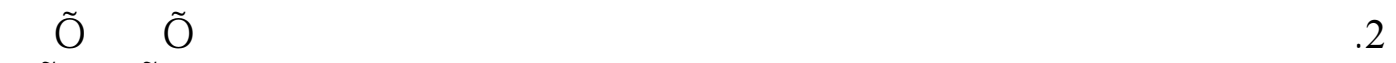
G) Ś̉ ř̀

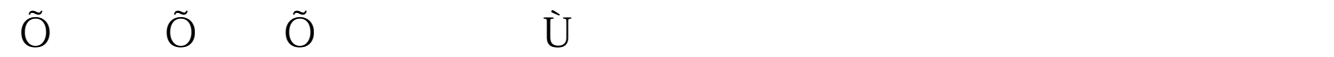

. ${ }^{[9]}$ řĐ̧Ủ 


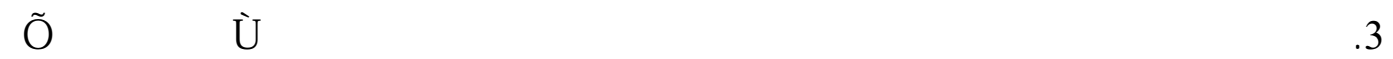

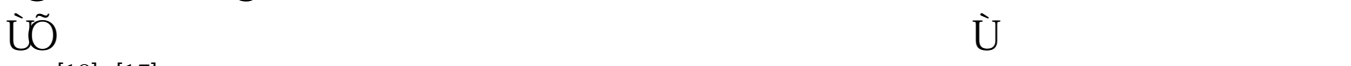

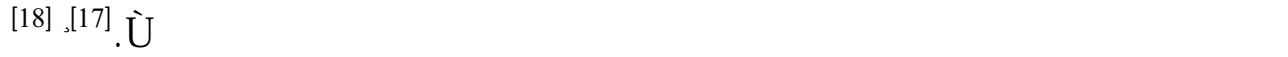

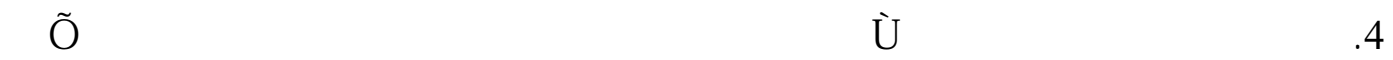

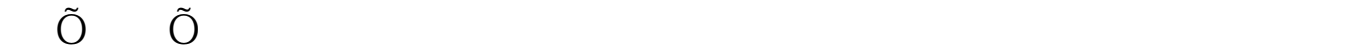
[20] [19][15]. r̀)

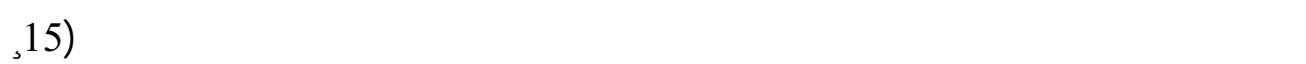

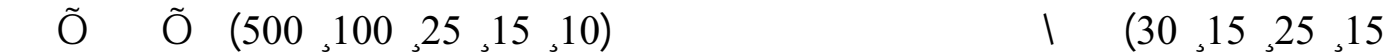

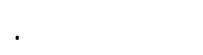

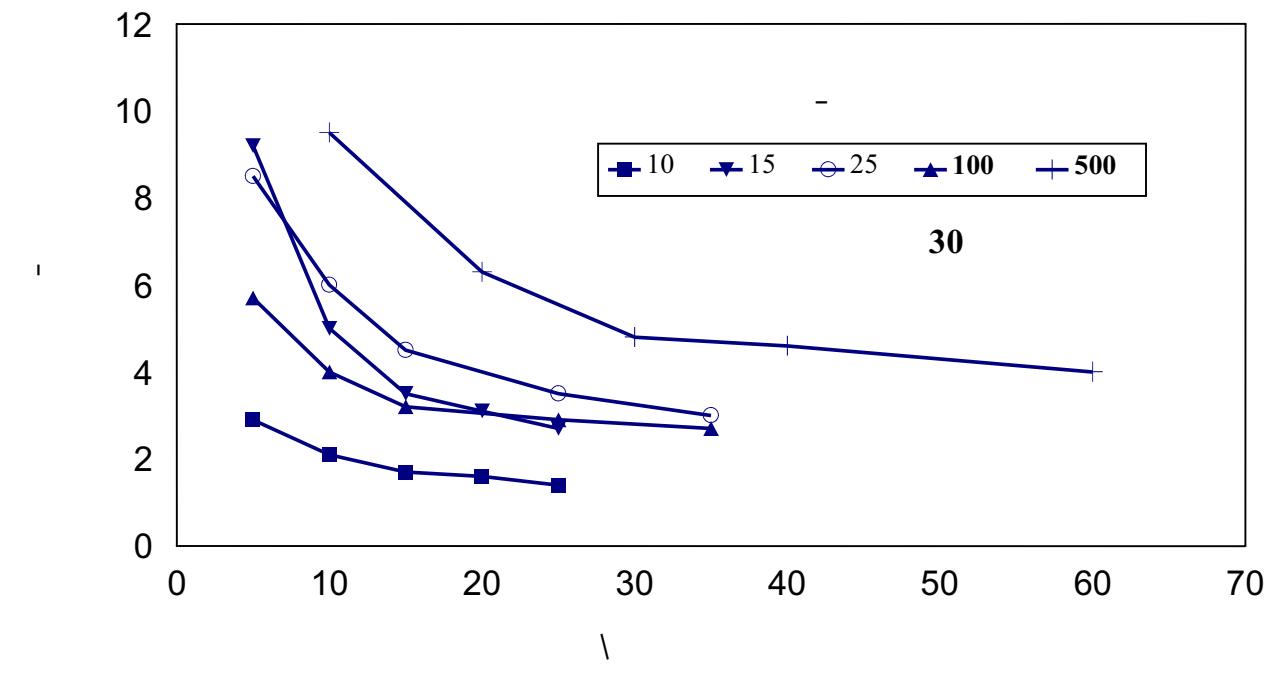

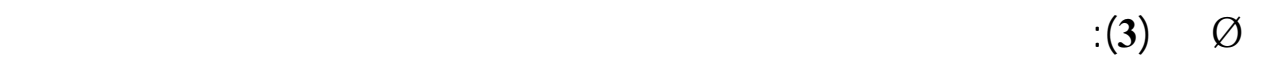

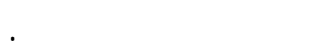

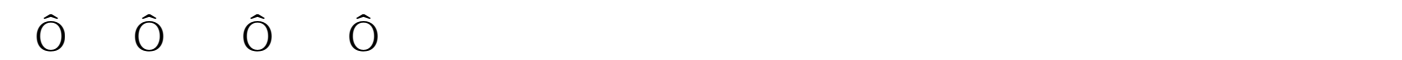

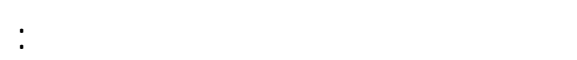

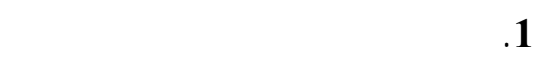

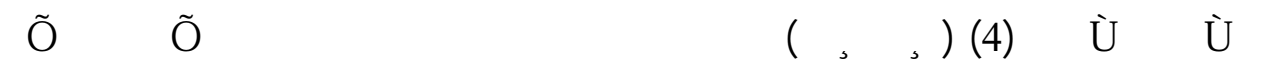

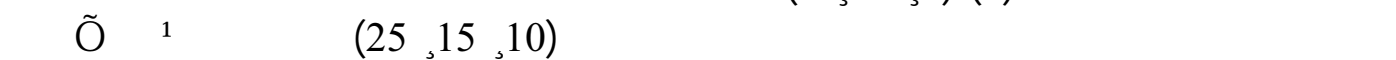
T» U

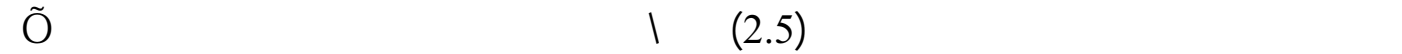

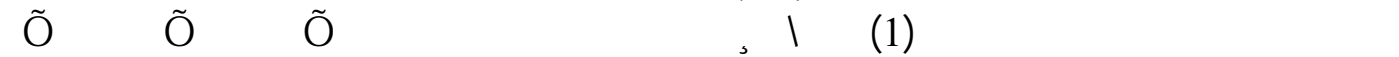

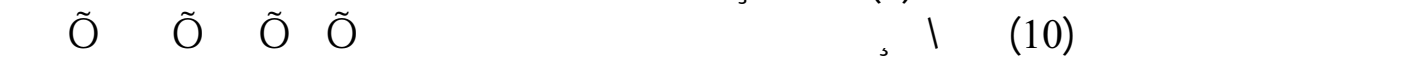

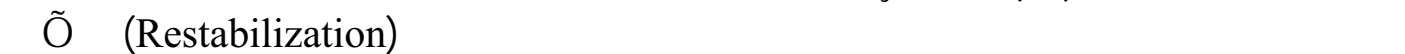

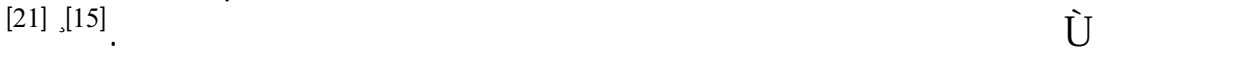

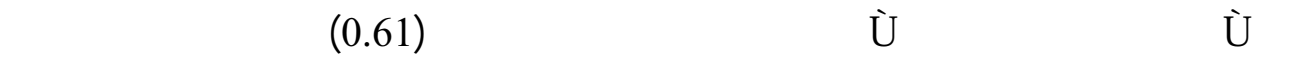

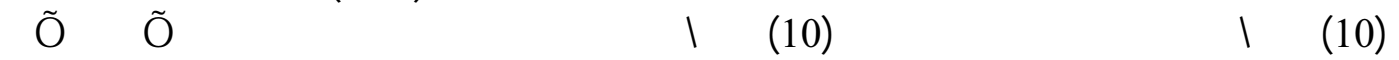

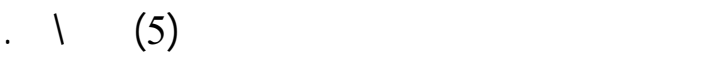



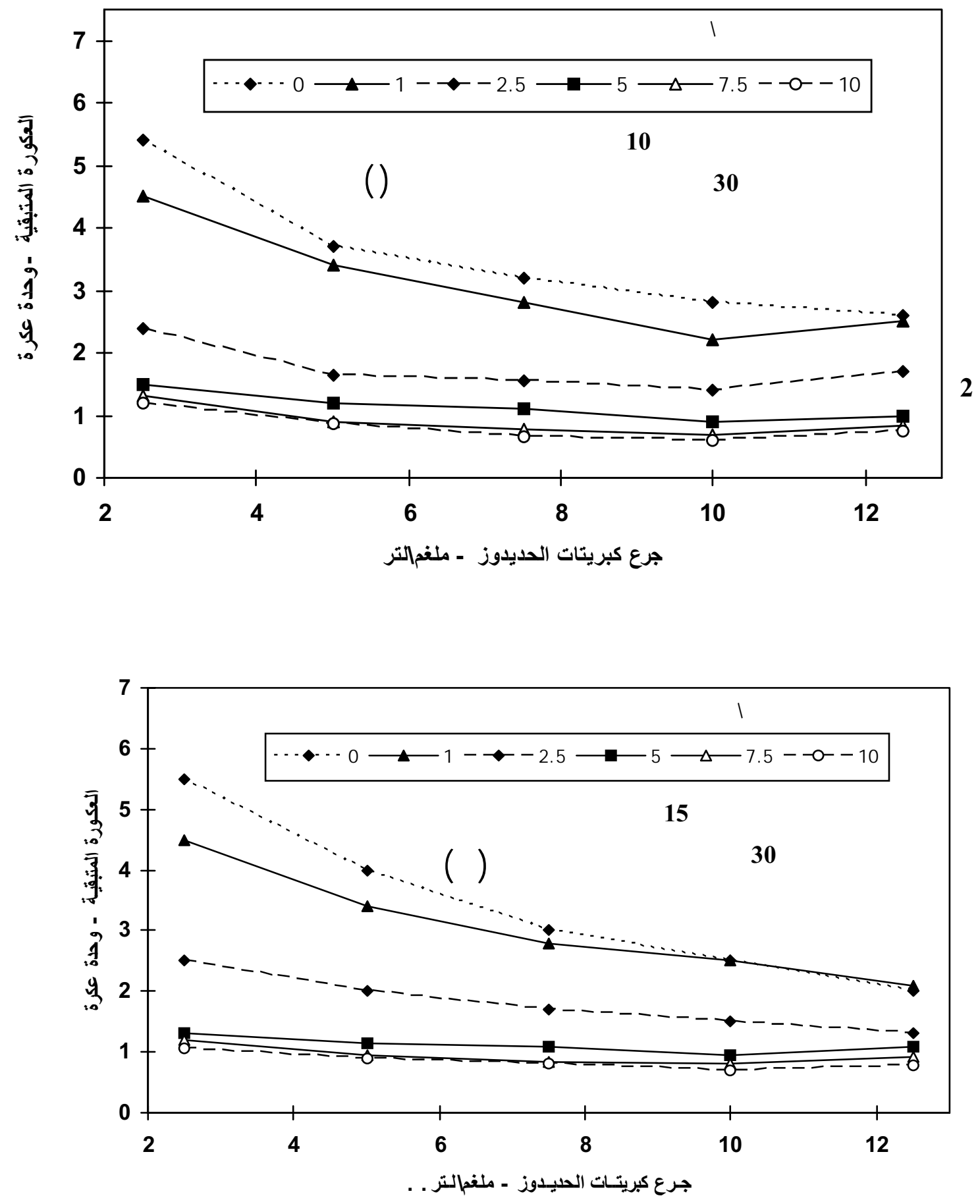


$\begin{array}{llll}\text { Al-Rafidain Engineering } & \text { Vol.14 } & \text { No.4 } & 2006\end{array}$

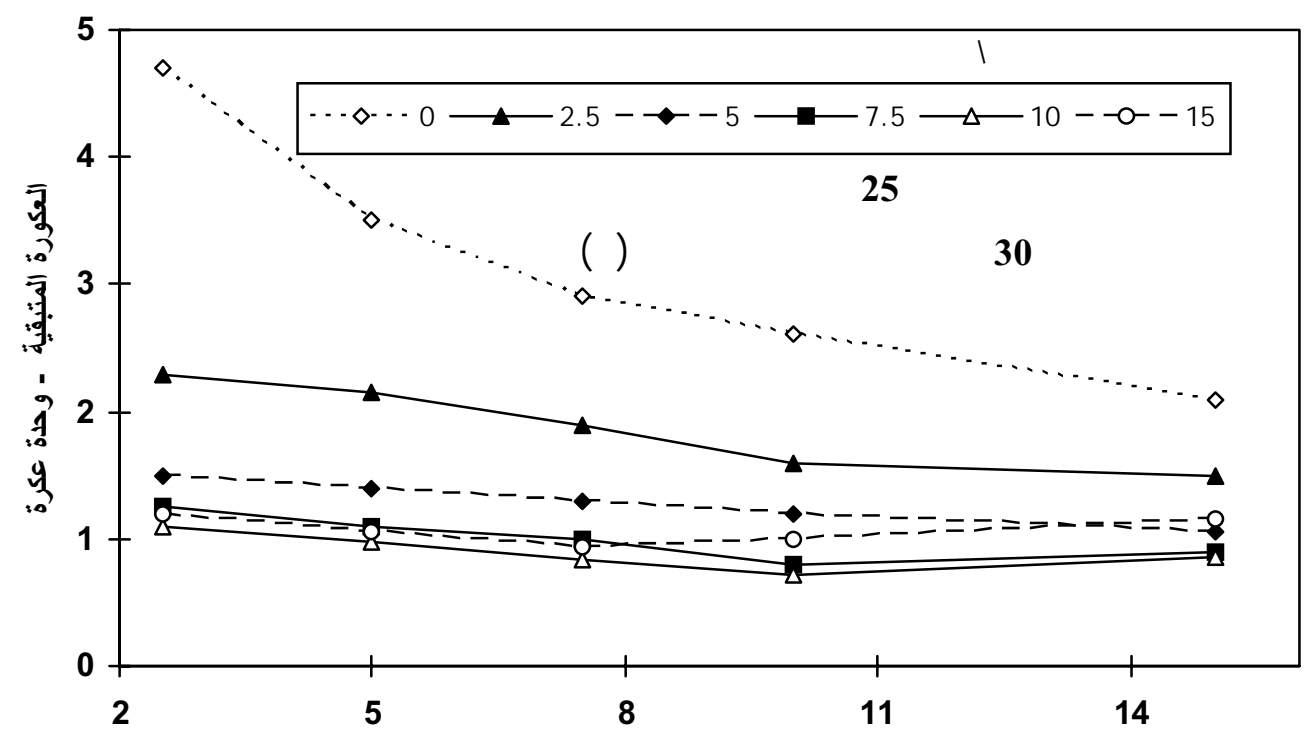

جرع كبريتات الحديدوز - ملغمالتر

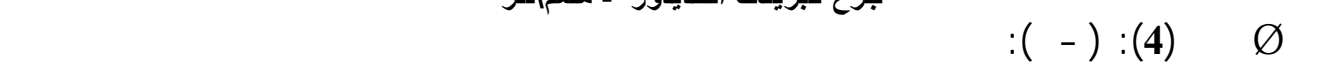

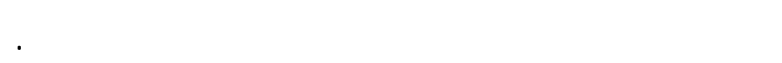

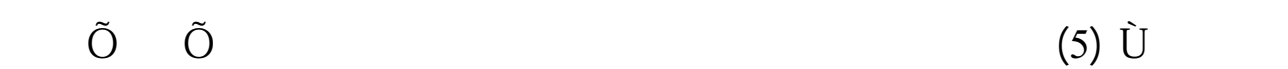

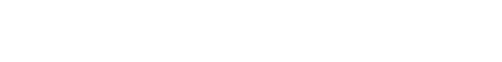

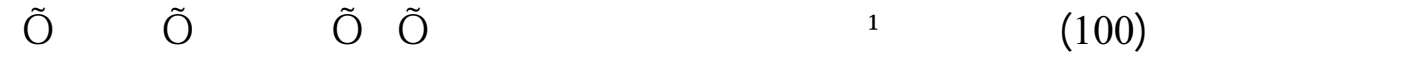

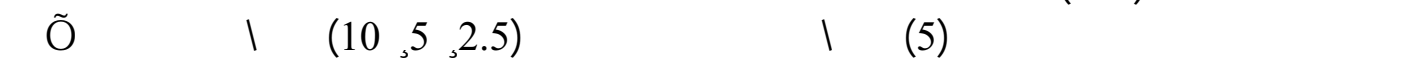

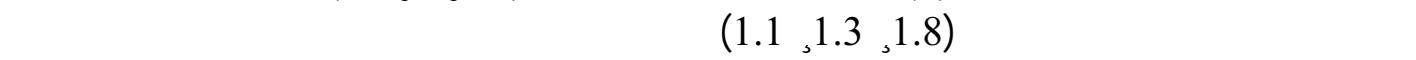

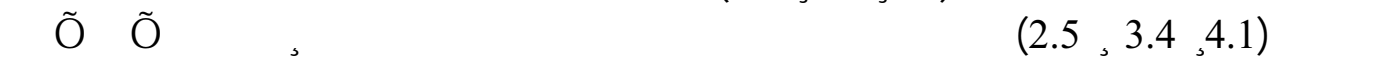

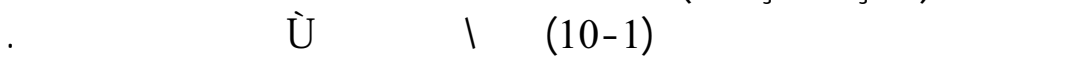

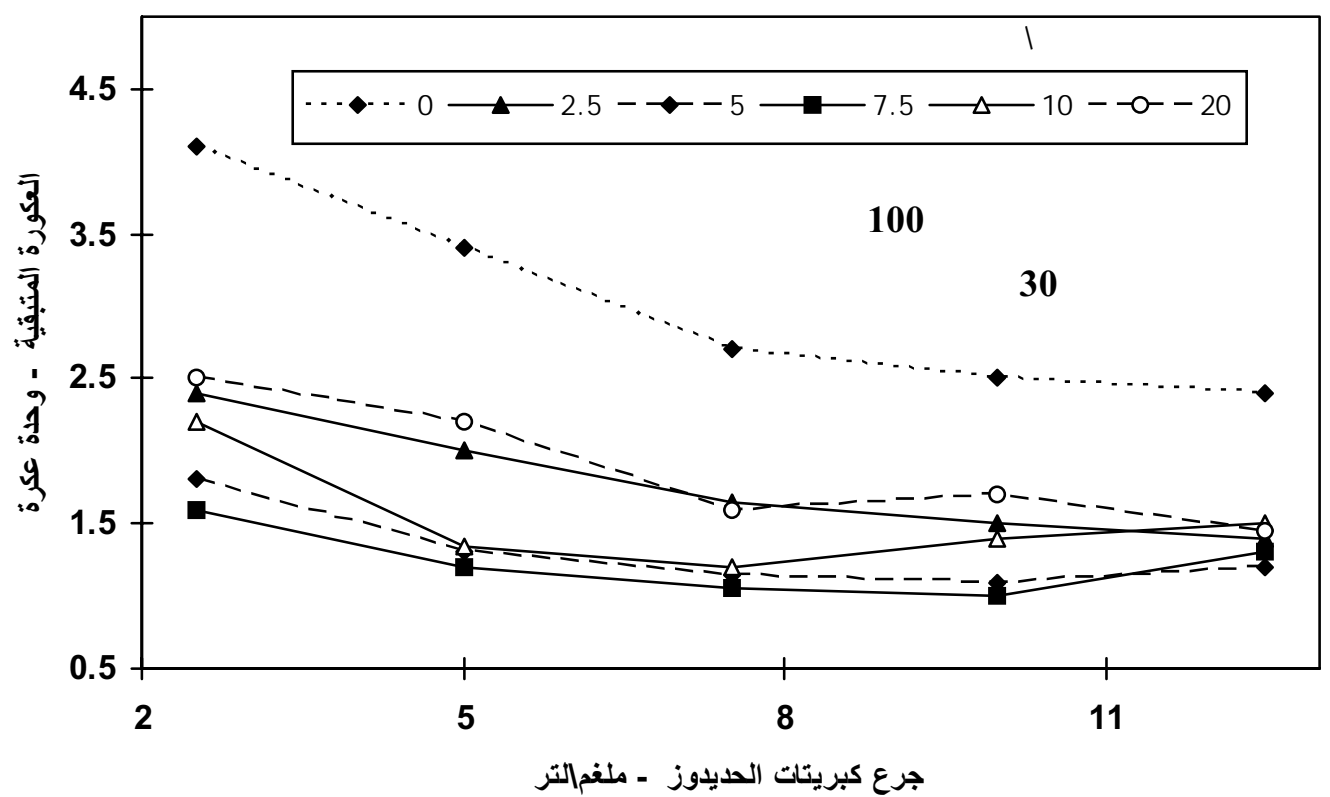

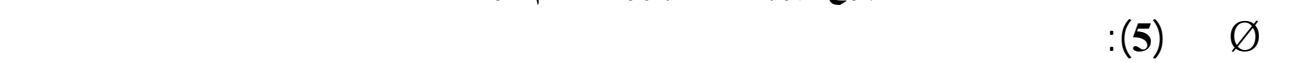

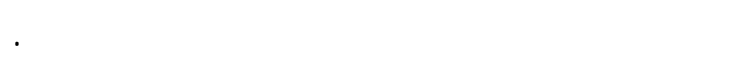




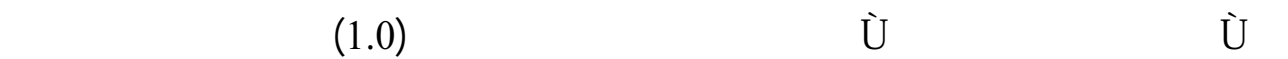

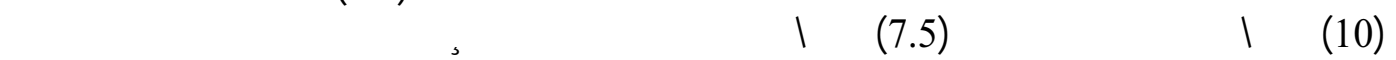

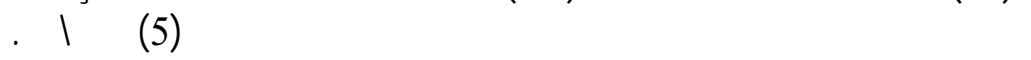

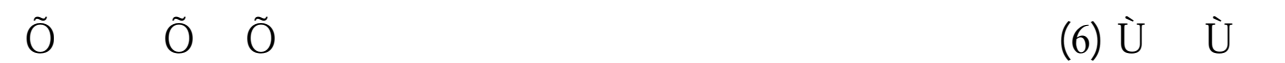

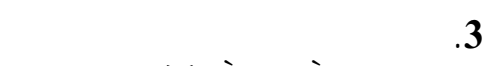

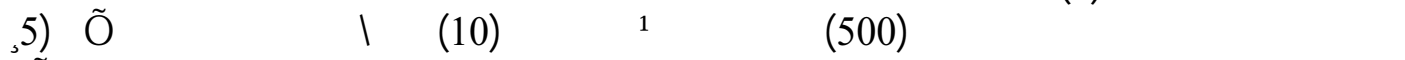

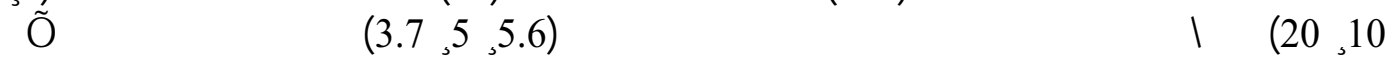
ũ fō ś

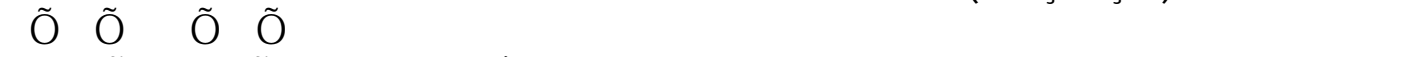

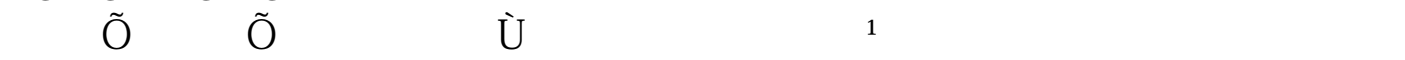

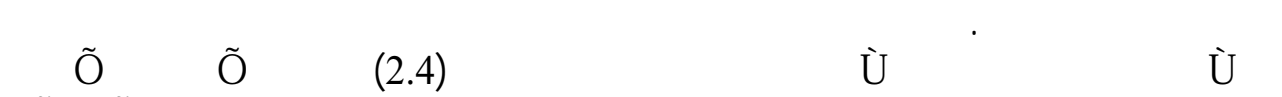

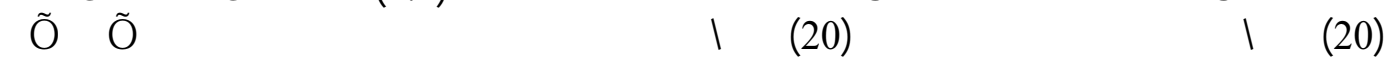

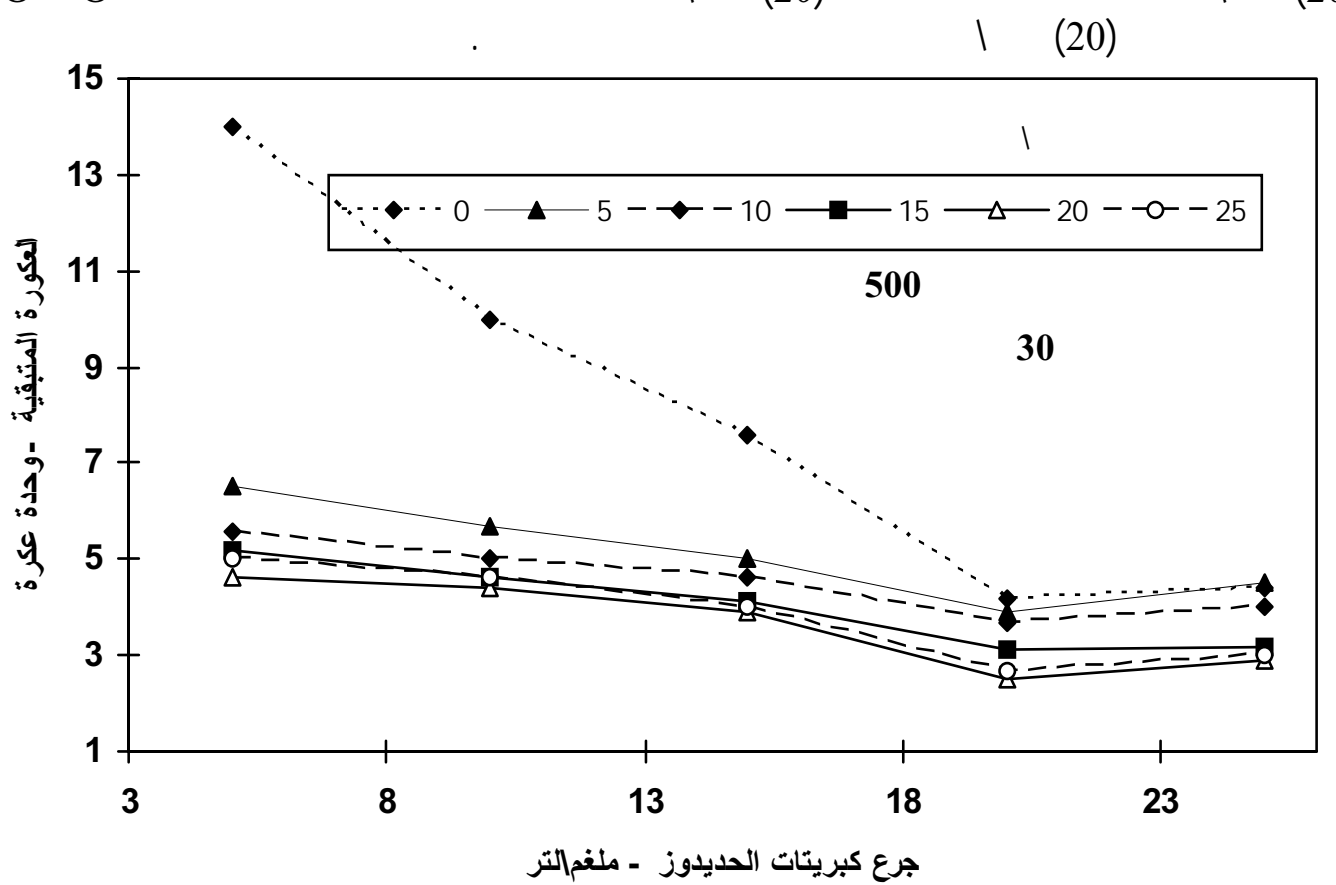

ũ)

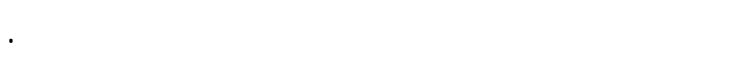

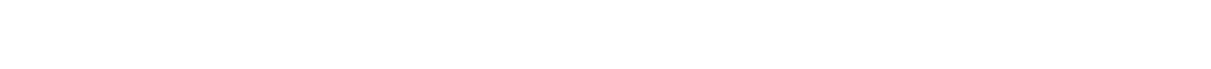

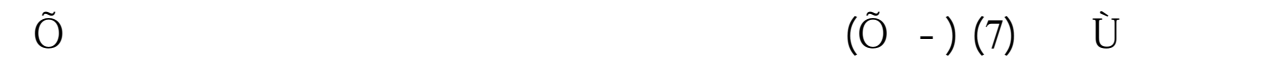

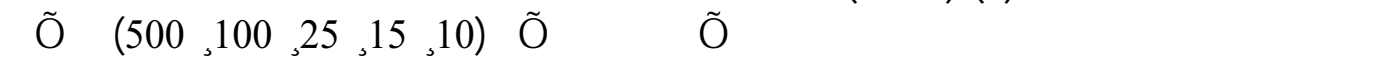

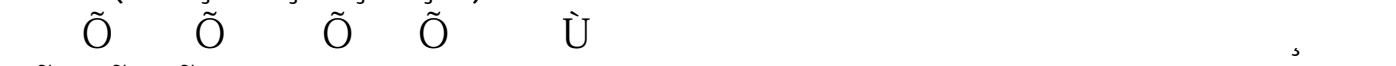

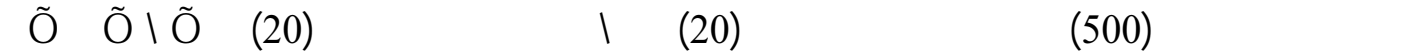

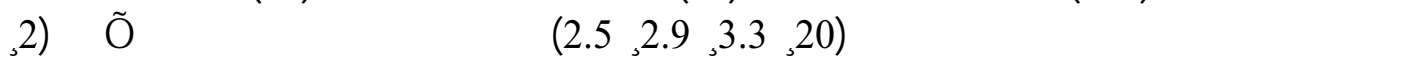

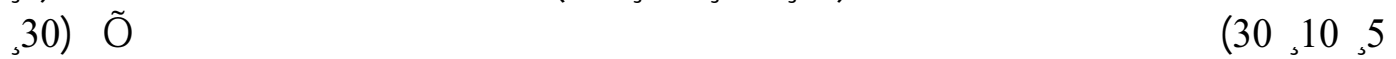

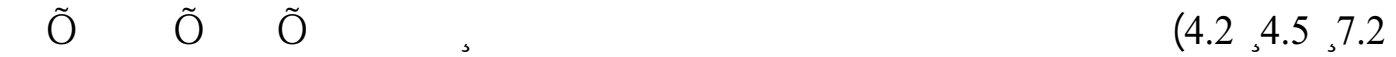

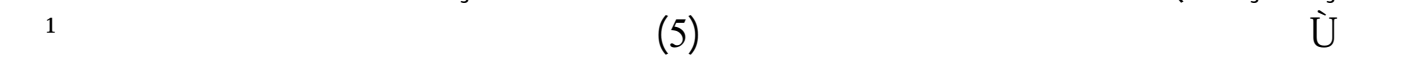

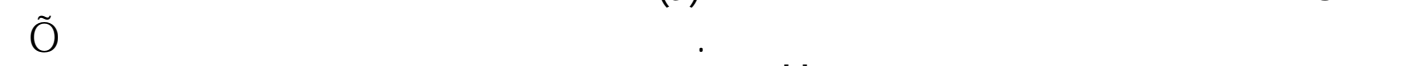

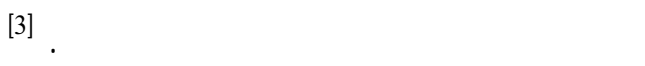




\begin{tabular}{llll} 
Al-Rafidain Engineering & Vol.14 & No.4 & 2006 \\
\hline
\end{tabular}

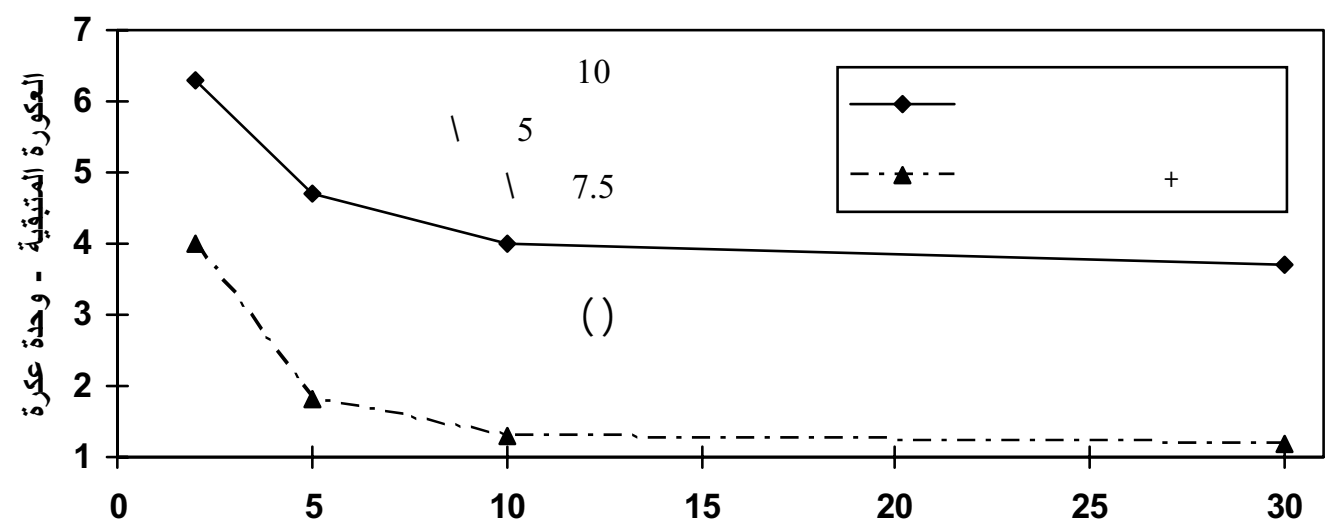

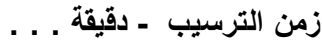
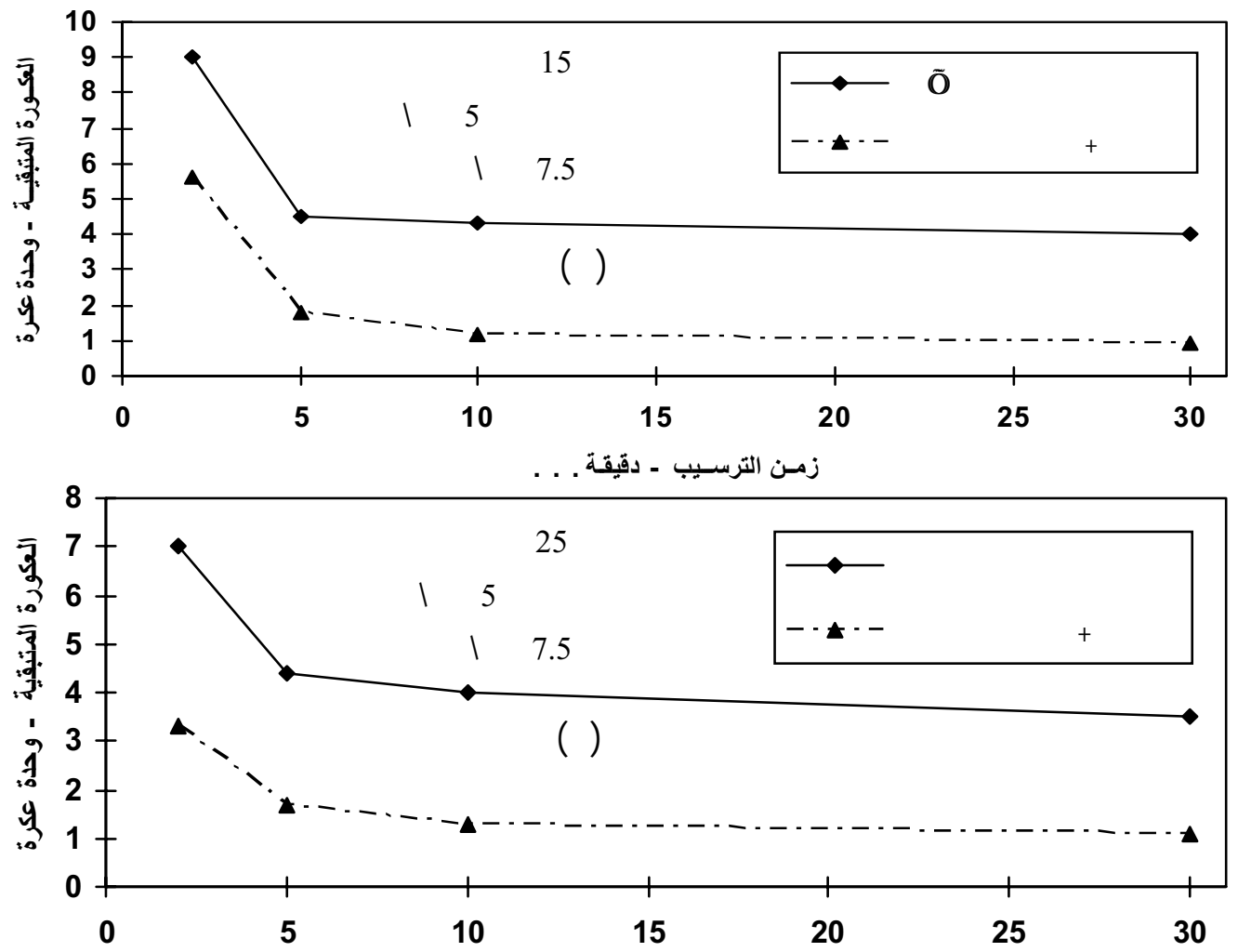

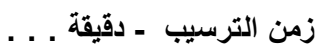

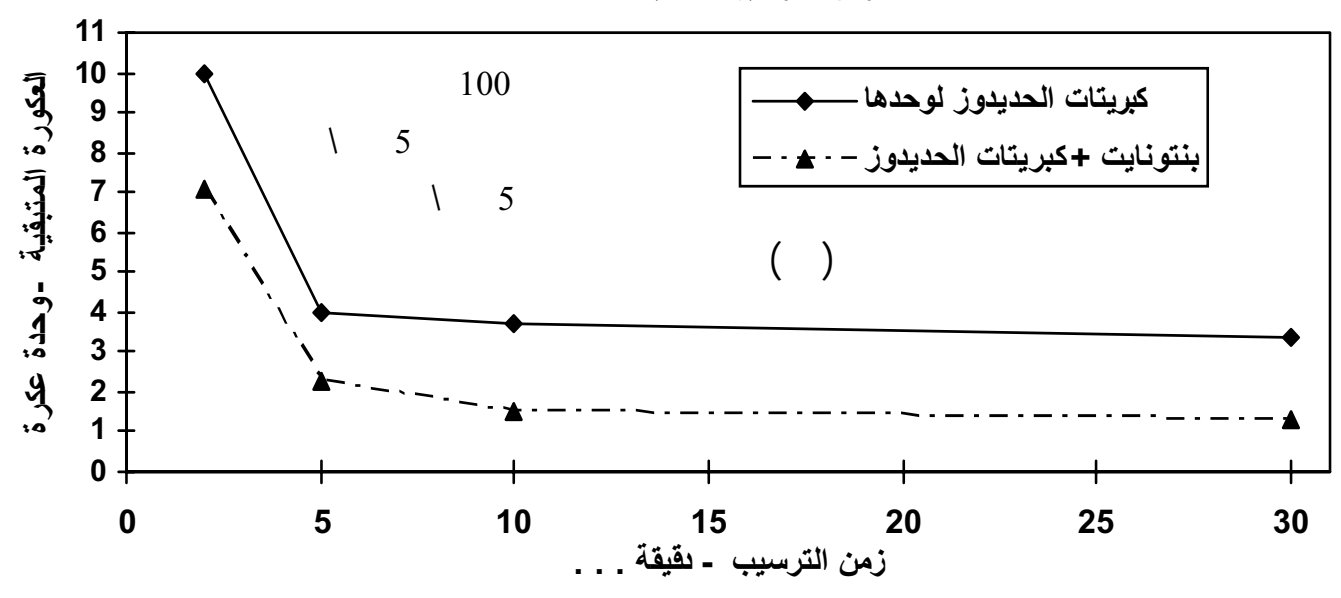




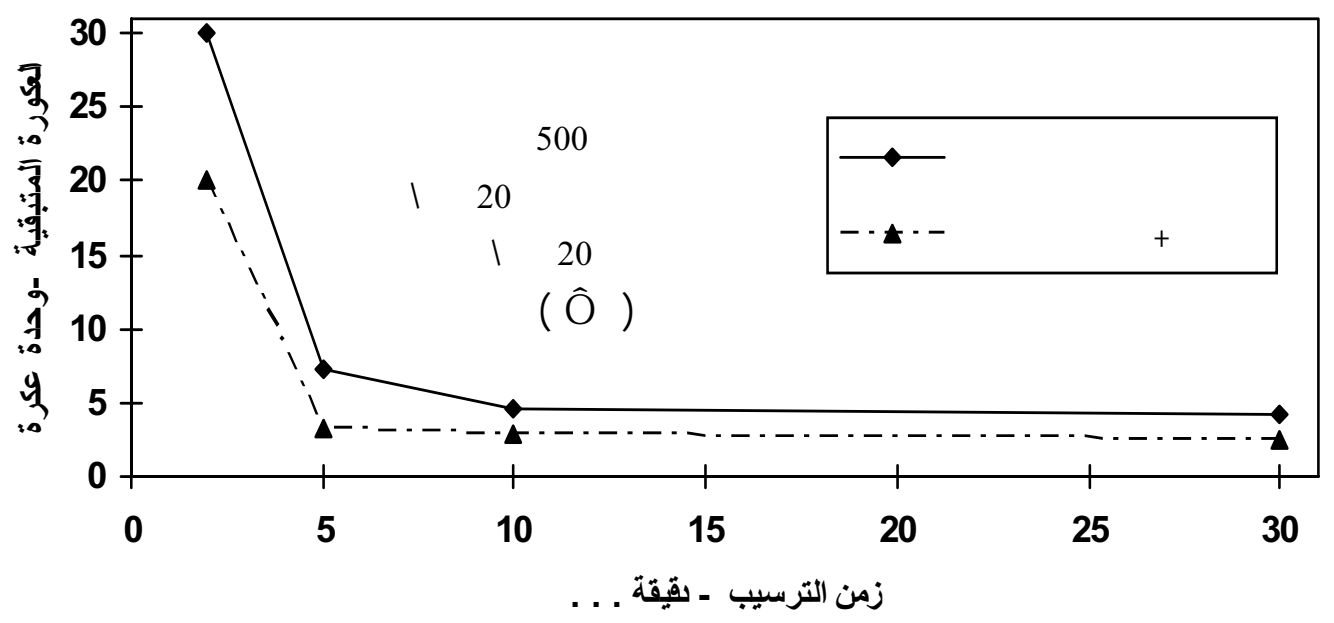

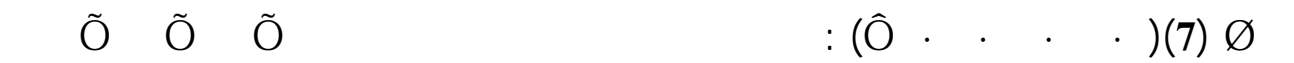

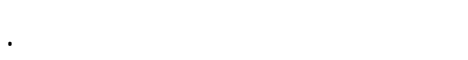

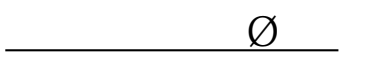

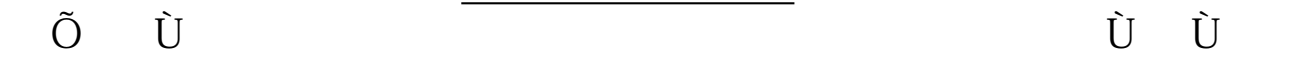

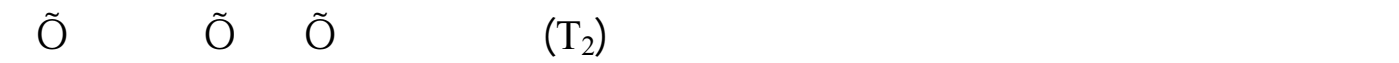

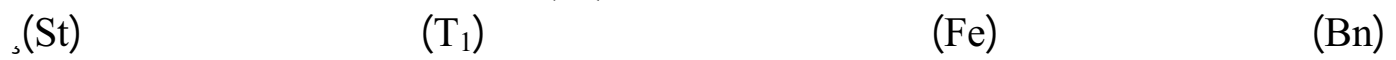

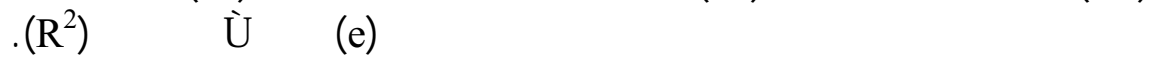

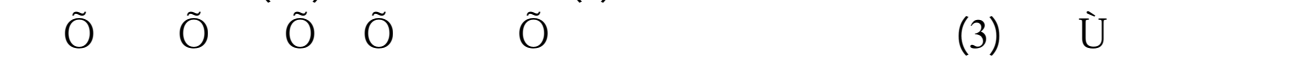

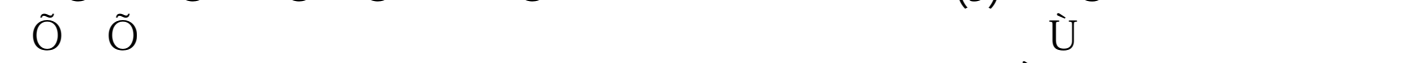

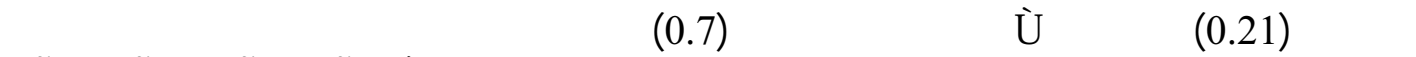

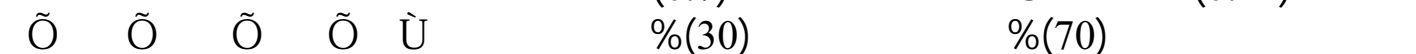

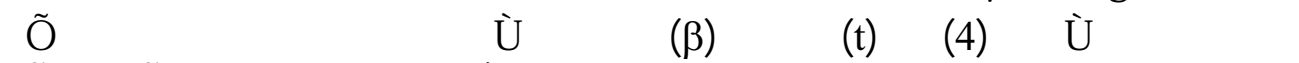

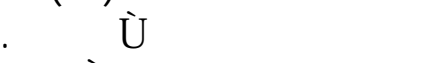

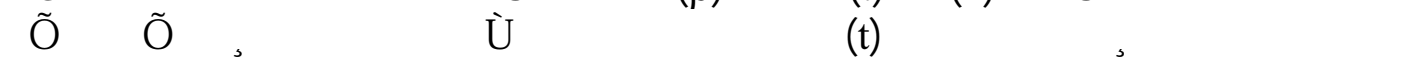
Rिग $Q$ F》

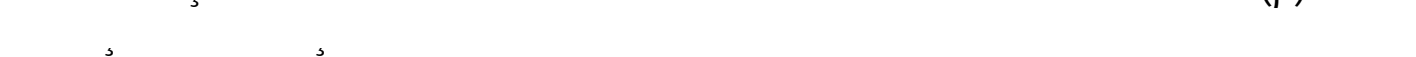

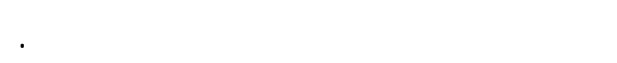

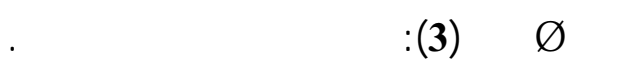

\begin{tabular}{|c|c|c|c|}
\hline 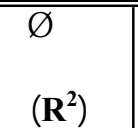 & 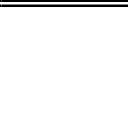 & 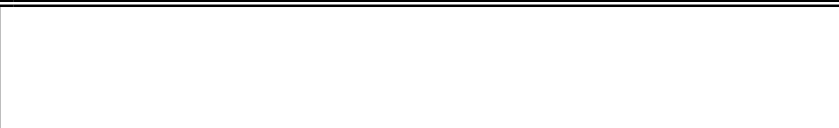 & 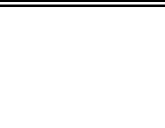 \\
\hline 0.308 & 9.54 & $\begin{aligned} \mathrm{T}_{2}= & 8.919-0.312 \mathrm{Fe}-0.447 \mathrm{Bn}-0.252 \mathrm{St}+ \\
& 0.041 \mathrm{~T}_{1}\end{aligned}$ & 1 \\
\hline 0.506 & 0.91 & $\begin{aligned} \sqrt{\mathrm{T}_{2}}= & 3.046-0.21 \sqrt{\mathrm{Fe}}-0.286 \sqrt{\mathrm{Bn}}-0.335 \\
& \sqrt{\mathrm{St}}+0.135 \sqrt{\mathrm{T}_{1}}\end{aligned}$ & 2 \\
\hline 0.7 & 0.21 & $\begin{aligned} \log \mathrm{T}_{2}= & 0.466-0.187 \log \mathrm{Fe}-0.276 \log \mathrm{Bn}- \\
& 0.499 \log \mathrm{St}+0.468 \log \mathrm{T}_{1}\end{aligned}$ & 3 \\
\hline
\end{tabular}




\begin{tabular}{llll} 
Al-Rafidain Engineering & Vol.14 & No.4 & 2006 \\
\hline
\end{tabular}

Ũż Ś

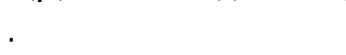

\begin{tabular}{|c|c|c|}
\hline \multicolumn{3}{|c|}{ 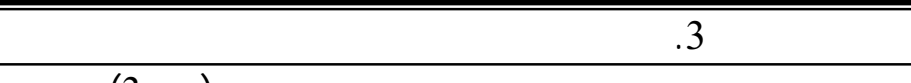 } \\
\hline & 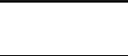 & LITITR \\
\hline t řç8 & $\beta \check{\text { řclB }}$ & 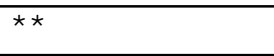 \\
\hline$* 25.8$ & 0.74 & $\mathrm{~T}_{1}$ \\
\hline$* 22.2-$ & 0.54 & St \\
\hline$* 10.2-$ & $0.28-$ & $\mathrm{Bn}$ \\
\hline$*_{5}-$ & $0.13-$ & $\mathrm{Fe}$ \\
\hline
\end{tabular}

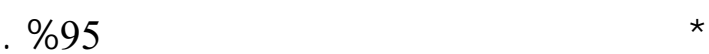

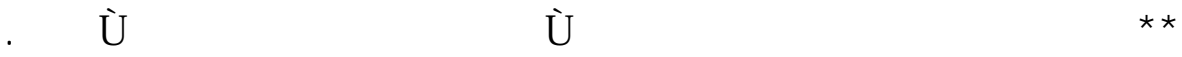

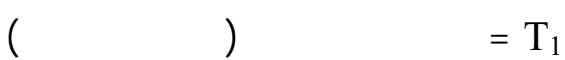

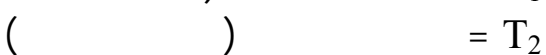

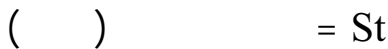

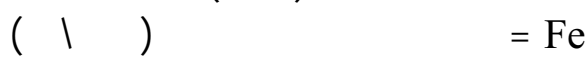

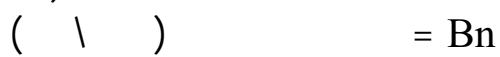

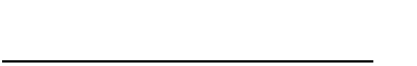

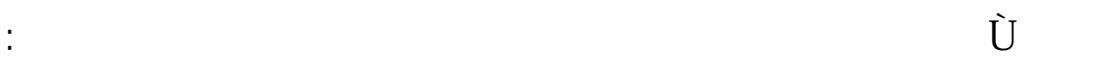

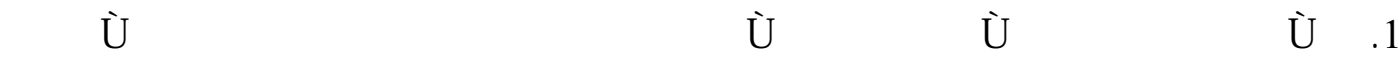
. ř

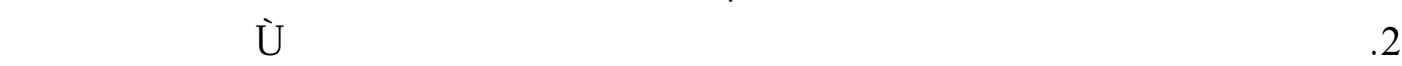

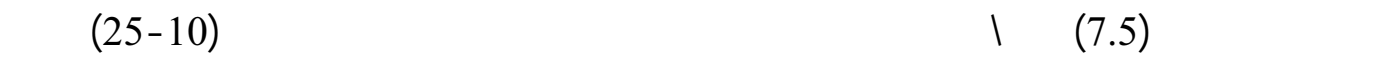

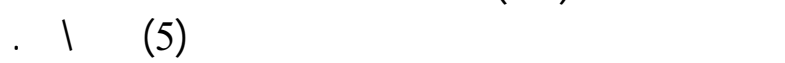

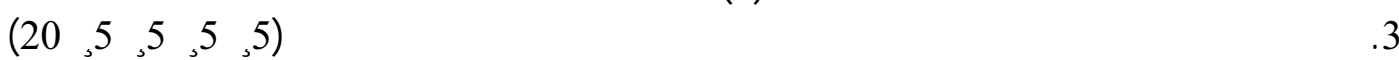

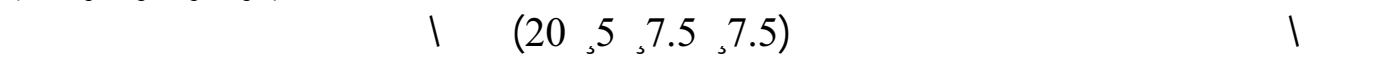

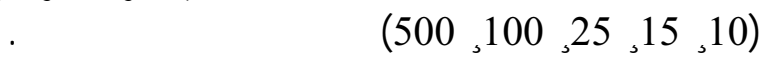

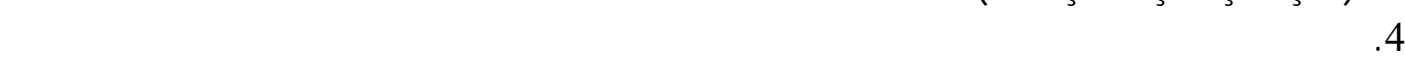

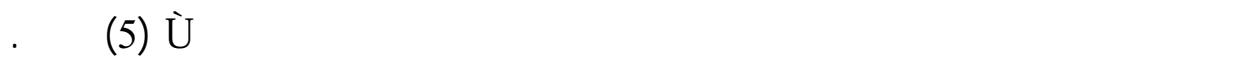
Śr

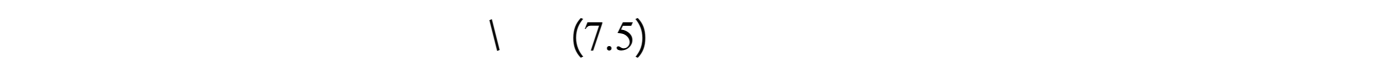

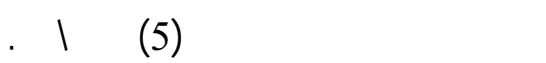

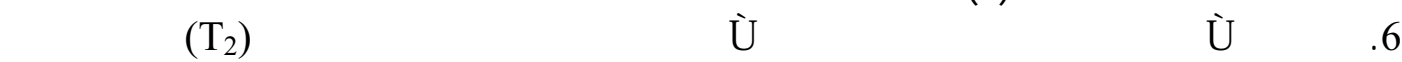
$\left.Q T_{1}\right)$ ry

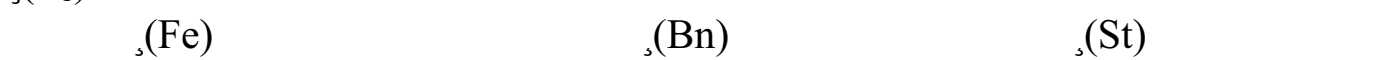

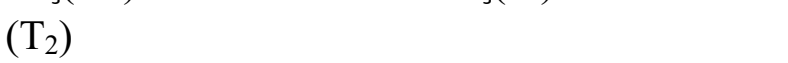

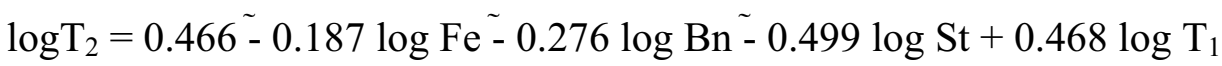

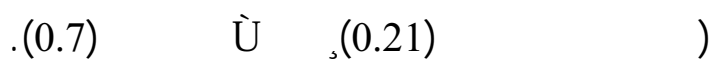

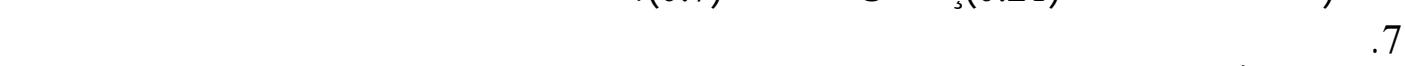

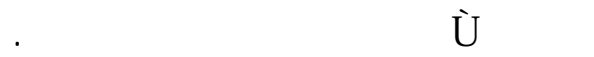

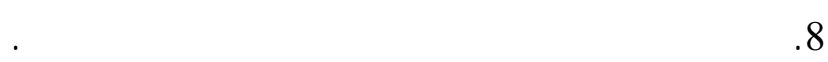




\section{etditisis}

1. Mahmmod, P.Y. and Ahmmad, F.H., 1985. A comparison between ferric sulfate and aluminum sulfate for clarification of Tigris river water in Mosul city, Iraqi conference on Engineering ICE 85,

2. Costello, J.J., 1984. Post precipitation in distribution system, Jour of AWWA, No.11.

3. Qureshi, N. and Malrnberg, R.J., 1985. Reducing aluminum residuals in finished water, jour of AWWA, Vol. 80, No 9.

4. Yasin, A. M., 1991, Effect of Using Lime in Conjunction with Alum and Chloride Coagulation on Turbidity and Hardness removal, M. Sc. Thesis, University of Mosul, Iraq.

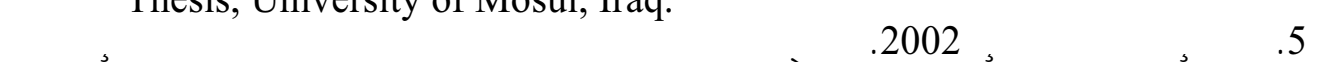

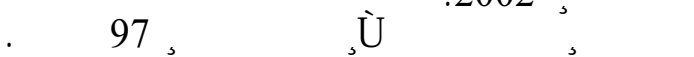

6. Matheson, D.T. and Forde, V.A., 1957. Survey of water purification practice in Canada, Jour of AWWA, Vol. 49, No. 12, 1522-1530.

7. Lund, E. and Nissen, B., 1986. Low technology water purification by bentonite clay flocculation as performed in sudanese villages vero logical examination, Water Research, Vol. 20, No. 1, 37-43.

8. York, D.W. and Drewry, A.W., 1974. Virus removal by chemical coagulation Jour. Of AWWA, Vol. 66, No. 12.

9. McCook, N.J. and West, J.R., 1978. The coagulation of a Kaolinite suspension with aluminum sulfate, Water Research, Vol. 12, No.10.

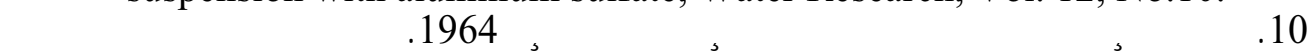

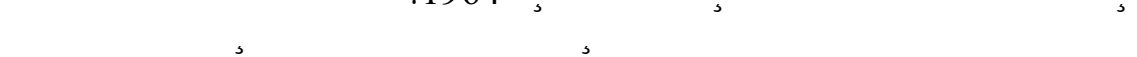

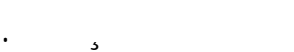

11. Al-Layla, M.A. and Middlebrooks, B.J., 1974. Optimum values for operational variables in turbidity removal, Water and Sewage Works, Vol. 121, No. 8.

12. Ammirtharajah, A. and Trusler, S.L., 1986. Destabilization of particles by turbulent rapid mixing, Jour of Environmental Engineering Division, ASCE, Vol. 112, No. 6.

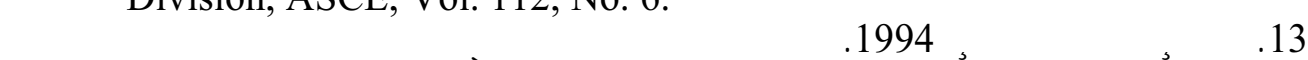

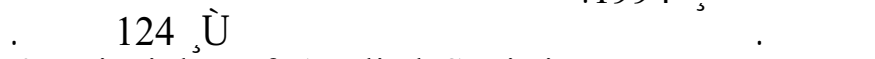

14. Melnyl, M, 1974. Principles of Applied Statistics, Pergamon press Inc., $619 \mathrm{p}$.

15. Ham, R.K. and Christman, R.F., 1969. Agglomerate size changes in coagulation, J. of sanitary Engineering Division, ASCE, Vol. 95, No. SA3.

16. Kim, W.; Ludwi, H.F. and Bishop, W.D., 1965. Cation-exchange capacity and $\mathrm{pH}$ in the coagulation process, Jour. of AWWA. 57(3), 327348.

17. Stumm, W. and Morgan, J.J., 1974. Aquatic chemistry, John Wiley and Sons, Inc.

18. Sawyer, G.N. and McCarty, P.L., 1978. Chemistry for environmental engineering, McGraw-Hill, LTD. USA, 532p.

19. Morris, J.K. and Knocke, W.F., 1984. Temperature effects on the use of metal-ion coagulants for water treatment, Jour of AWWA, Vol. 76, No. 74.

20. Kawamura, S 1991. Effectiveness of natural polyelectrolytes in water treatment, Jour of AWWA, Vol. 83, no. 10, 88-91.

21. Dentel, S.K. and Gosset, J.M., 1988. Mechanisms of coagulation with aluminum salts, Jour of AWWA, Vol. 80 ,No. 9187-198. 